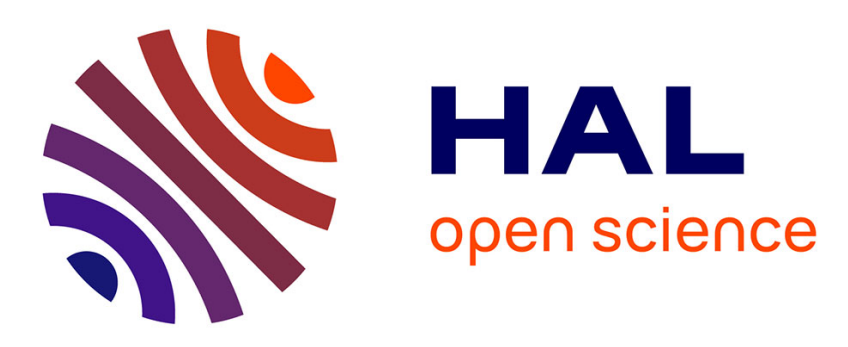

\title{
Operation modes and workspace of a 4-rRUU Parallel Manipulator
}

\author{
Abhilash Nayak, Stéphane Caro, Philippe Wenger
}

\section{To cite this version:}

Abhilash Nayak, Stéphane Caro, Philippe Wenger. Operation modes and workspace of a 4-rRUU Parallel Manipulator. In: Uhl T. (eds) Advances in Mechanism and Machine Science. IFToMM WC 2019. Mechanisms and Machine Science, vol 73. Springer, Cham, pp.649-657, 2019, 10.1007/978-3030-20131-9_65. hal-02404455

\section{HAL Id: hal-02404455 https://hal.science/hal-02404455}

Submitted on 11 Dec 2019

HAL is a multi-disciplinary open access archive for the deposit and dissemination of scientific research documents, whether they are published or not. The documents may come from teaching and research institutions in France or abroad, or from public or private research centers.
L'archive ouverte pluridisciplinaire HAL, est destinée au dépôt et à la diffusion de documents scientifiques de niveau recherche, publiés ou non, émanant des établissements d'enseignement et de recherche français ou étrangers, des laboratoires publics ou privés. 


\title{
Operation modes and workspace of a 4-rRUU Parallel Manipulator
}

\author{
Abhilash Nayak ${ }^{1}$, Stéphane Caro $^{2}$, and Philippe Wenger ${ }^{2}$ \\ 1 Centrale Nantes, Laboratoire des Sciences du Numerique de Nantes (LS2N), \\ 2 CNRS, Laboratoire des Sciences du Numerique de Nantes (LS2N), \\ \{abhilash.nayak, stephane.caro, philippe.wenger\}@ls2n.fr
}

\begin{abstract}
A lower-mobility parallel manipulator with multiple operation modes can be considered as inherently reconfigurable. This is the case for 4-RUU parallel manipulators that have three different operation modes. Allowing the first revolute joint axis to have any horizontal orientation leads to a dual reconfigurable 4-rRUU mechanism. This paper presents an interpolation approach to determine its operation modes as functions of the orientation of its base revolute joint axes. Furthermore, its limb singularities are characterized by differentiating the constraint equations with respect to the actuated joint variables which are further used to visualize its translational workspace for three mutually perpendicular orientations of its base revolute joint axes.
\end{abstract}

Keywords: parallel manipulator, dual reconfigurable, 4-RUU, operation modes, workspace

\section{Introduction}

Different configuration space regions of a parallel manipulator (PM) with possibly different type or number of degrees of freedom are known as the operation modes. They were first observed for the 3-URU DYMO robot by Zlatanov et al. [1] showing that it exhibits five different types of platform motion. A complete characterization of operation modes for various PMs using Study's kinematic mapping and algebraic geometry tools has been accomplished in [2-4]. Moreover, the operation modes of a much more complicated 4-RUU PM with vertical R-joint axes were determined by splitting it into two 2-RUU PMs [5]. Additionally, a novel dual reconfigurable 4-rRUU PM was proposed, constituting a double-Hooke's joint in each limb to reconfigure the R-joint axes to have any horizontal orientations. Its operation modes were determined for some specific configurations [6]. This paper proposes an interpolation approach to determine the operation modes as a function of the orientation of base R-joint axes. Furthermore, its workspace is plotted, thanks to the algebraic expression of its limb singularities.

The organization of the paper is as follows: Section 2 presents the architecture of the dual reconfigurable 4-rRUU PM. Section 3 deals with the derivation of constraint equations and the determination of operation modes of the 4-rRUU 
PM in terms of orientations of the base revolute joint axes. Section 4 shows the translational workspace when all the base R-joints are oriented in the same direction.

\section{Manipulator architecture}

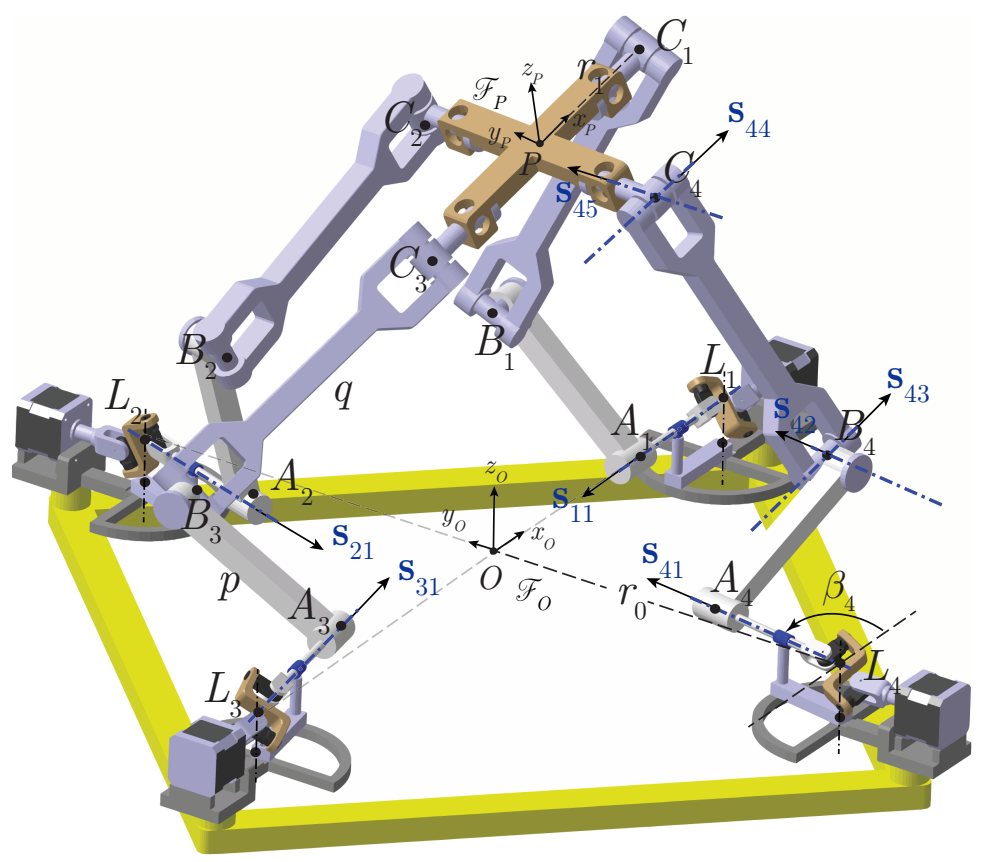

Fig. 1: A 4-rRUU parallel manipulator

The dual reconfigurable 4-rRUUPM with a square base and a platform is shown in Fig. 1. Each limb consists of a double Hooke's joint (rR) followed by two universal joints (UU). Point $L_{i}, i=1,2,3,4$ is the intersection point of the pivotal axis of the double-Hooke's joint linkage and the first revolute joint axis. Point $A_{i}$ lies on the first revolute joint axis of the PM. Points $B_{i}$ and $C_{i}$ are the geometric centers of the first and the second universal joints, respectively. Points $L_{i}$ and $C_{i}$ are the vertices of the square base and the platform, respectively. $\mathscr{F}_{O}$ and $\mathscr{F}_{P}$ are the coordinate frames attached to the fixed base and the moving platform such that their origins $O$ and $P$ lie at the centers of the respective squares. The circum-radii of the base and platform squares are denoted $r_{0}$ and $r_{1}$, respectively. $p$ and $q$ are the link lengths. The revolute-joint axes vectors in $i$-th limb are marked $\mathbf{s}_{i j}, i=1,2,3,4 ; j=1, \ldots, 5$. Vectors $\mathbf{s}_{i 1}$ and $\mathbf{s}_{i 2}$ are always parallel, so are vectors $\mathbf{s}_{i 3}$ and $\mathbf{s}_{i 4}$. For simplicity, it is assumed that the 
orientation of vector $\mathbf{s}_{i 1}$ expressed in coordinate frame $\mathscr{F}_{O}$ is the same as that of $\mathbf{s}_{i 5}$ expressed in coordinate frame $\mathscr{F}_{P}$.

\section{Operation Mode Analysis}

\subsection{Constraint Equations}

Since the reconfigurable revolute joint is actuated, a RUU limb must satisfy the following two constraints:

1. The second revolute joint axis, the fifth revolute joint axis and link $B C$ must lie in the same plane. In other words, the scalar triple product of the corresponding vectors must be null:

$$
g_{i}:\left(\mathbf{b}_{i}-\mathbf{c}_{i}\right)^{T}\left(\mathbf{s}_{i 2} \times \mathbf{s}_{i 5}\right)=0, \quad i=1,2,3,4
$$

2. The length of link $B C$ must be $q$ :

$$
g_{i+4}:\left\|\mathbf{b}_{i}-\mathbf{c}_{i}\right\|-q=0, \quad i=1,2,3,4
$$

Since the length of link $B C$ does not affect the operation modes of the 4-rRUU PM, only the principal geometric constraint from Eq. (1) is considered. To express it algebraically, the homogeneous coordinates of the necessary vectors expressed in frame $\mathcal{F}_{O}$ are listed below:

$$
\begin{aligned}
& { }^{0} \mathbf{l}_{i}=\mathbf{R}_{z}\left(\lambda_{i}\right)\left[1, r_{0}, 0,0\right]^{T} \\
& { }^{0} \mathbf{a}_{i}={ }^{0} \mathbf{l}_{i}+\mathbf{R}_{z}\left(\lambda_{i}+\beta_{i}\right)\left[0,0, l_{i}, 0\right]^{T} \\
& { }^{0} \mathbf{b}_{i}={ }^{0} \mathbf{a}_{i}+\mathbf{R}_{z}\left(\lambda_{i}+\beta_{i}\right)\left[0, p \cos \left(\theta_{i}\right), 0, p \sin \left(\theta_{i}\right)\right]^{T} \\
& { }^{0} \mathbf{c}_{i}=\mathbf{F} \mathbf{R}_{z}\left(\lambda_{i}\right)\left[1, r_{1}, 0,0\right]^{T}, \\
& { }^{0} \mathbf{s}_{i 2}=\mathbf{R}_{z}\left(\lambda_{i}+\beta_{i}\right)[0,0,1,0]^{T}, \\
& { }^{0} \mathbf{s}_{i 5}=\mathbf{F} \mathbf{R}_{z}\left(\lambda_{i}+\beta_{i}\right)[0,0,1,0]^{T}, \quad i=1,2,3,4 .
\end{aligned}
$$

where $l_{i}=\left|A_{i} L_{i}\right|, \mathbf{R}_{z}(\cdot)$ is the homogeneous rotation matrix about the $z$-axis, $\lambda_{i}$ for the $i$-th limb is given by $\lambda_{1}=0, \lambda_{2}=\frac{\pi}{2}, \lambda_{3}=\pi, \lambda_{4}=\frac{3 \pi}{2}$ and $\theta_{i}$ is the actuated joint angle. $\mathbf{F}$ is the following transformation matrix:

$\mathbf{F}=\frac{1}{\Delta}\left[\begin{array}{cccc}\Delta & 0 & 0 & 0 \\ d_{1} x_{0}{ }^{2}+x_{1}{ }^{2}-x_{2}{ }^{2}-x_{3}{ }^{2} & -2 x_{0} x_{3}+2 x_{1} x_{2} & 2 x_{0} x_{2}+2 x_{1} x_{3} \\ d_{2} & 2 x_{0} x_{3}+2 x_{1} x_{2} & x_{0}{ }^{2}-x_{1}{ }^{2}+x_{2}{ }^{2}-x_{3}{ }^{2} & -2 x_{0} x_{1}+2 x_{2} x_{3} \\ d_{3} & -2 x_{0} x_{2}+2 x_{1} x_{3} & 2 x_{0} x_{1}+2 x_{2} x_{3} & x_{0}{ }^{2}-x_{1}{ }^{2}-x_{2}{ }^{2}+x_{3}{ }^{2}\end{array}\right]$

with $\Delta=x_{0}^{2}+x_{1}^{2}+x_{2}^{2}+x_{3}^{2} \neq 0$

and $d_{1}=-2 x_{0} y_{1}+2 x_{1} y_{0}-2 x_{2} y_{3}+2 x_{3} y_{2}$, 


$$
\begin{aligned}
& d_{2}=-2 x_{0} y_{2}+2 x_{1} y_{3}+2 x_{2} y_{0}-2 x_{3} y_{1}, \\
& d_{3}=-2 x_{0} y_{3}-2 x_{1} y_{2}+2 x_{2} y_{1}+2 x_{3} y_{0},
\end{aligned}
$$

where $x_{j}, y_{j}, j=0,1,2,3$ are called Study parameters of the transformation $\mathbf{F}$. A point $P=\left(x_{0}: x_{1}: x_{2}: x_{3}: y_{0}: y_{1}: y_{2}: y_{3}\right) \in \mathbb{P}^{7}$ represents an Euclidean transformation, if and only if $P$ lies in a 6 -dimensional quadric, $S_{6}^{2} \in \mathbb{P}^{7}$ called the Study quadric:

$$
S_{6}^{2}: x_{0} y_{0}+x_{1} y_{1}+x_{2} y_{2}+x_{3} y_{3}=0
$$

Thus, Eq. (1) is derived for each limb algebraically by substituting $t_{i}=\tan \left(\frac{\theta_{i}}{2}\right)$ and $v_{i}=\tan \left(\frac{\beta_{i}}{2}\right), i=1,2,3,4$. The equations are not displayed here due to space limits.

The constraint polynomials $g_{i}, i=1,2,3,4$ along with Study's quadric form the following ideal ${ }^{3}$ :

$$
\mathcal{I}=\left\langle g_{1}, g_{2}, g_{3}, g_{4}, \mathrm{~S}_{6}^{2}\right\rangle \subseteq k\left[x_{0}, x_{1}, x_{2}, x_{3}, y_{0}, y_{1}, y_{2}, y_{3}\right]
$$

To simplify the determination of the operation modes, the 4-rRUU PM is split into two 2-rRUU PMs [5] by considering two ideals:

$$
\begin{aligned}
\mathcal{I}_{(I)} & =\left\langle g_{1}, g_{3}, \mathrm{~S}_{6}^{2}\right\rangle \\
\mathcal{I}_{(I I)} & =\left\langle g_{2}, g_{4}, \mathrm{~S}_{6}^{2}\right\rangle
\end{aligned}
$$

Furthermore, $\mathcal{I}_{(I)}$ and $\mathcal{I}_{(I I)}$ can be decomposed into simpler ideals using primary decomposition to understand the operation modes of the 2-rRUU PMs. Thus, the union of the corresponding prime ideals characterize the operation modes of the whole 4-rRUU PM. Two cases can be considered:

Case 1: When the revolute joint axes are arbitrarily oriented, the primary decomposition of $\mathcal{I}_{(I)}$ and $\mathcal{I}_{(I I)}$ leads to one sub-ideal each. These sub-ideals depend on the design parameters and are mixed motion modes which are not of interest in the context of this paper.

Case 2: When the opposite revolute joint axes have the same orientation i.e. $v_{1}=v_{3}$ and $v_{2}=v_{4}$, the operation modes can be determined as follows:

All planar orientations of the $r R$-joint axes are covered by varying $\beta_{i} \in\left[-90^{\circ}, 90^{\circ}\right]$ and hence $v_{i} \in[-1,1]$. Design parameters were substituted as $r_{0}=2, r_{1}=3, p=$ $5, q=7$ to simplify the primary decomposition of ideals $\mathcal{I}_{(I)}$ and $\mathcal{I}_{(I I)}$ in Eq. (7). The operation modes could be determined only when arbitrary rational values are substituted for $v_{1}=v_{3}=v_{13}$ and $v_{2}=v_{4}=v_{24}$. The primary decomposition is performed in a computer algebra system Singular and it leads to three subideals each. The first two are independent of the design parameters and actuated

\footnotetext{
3 The ideal generated by the given polynomials is the set of all combinations of these polynomials using coefficients from the polynomial ring $k\left[x_{0}, x_{1}, x_{2}, x_{3}, y_{0}, y_{1}, y_{2}, y_{3}\right][7]$.
} 
variables. They are of the following form:

$$
\begin{gathered}
\mathcal{I}_{(I)}=\mathcal{I}_{1(I)} \cap \mathcal{I}_{2(I)} \cap \mathcal{I}_{3(I)}, \\
\text { where } \mathcal{I}_{1(I)}=\left\langle x_{0}, h_{1} x_{1}+h_{2} x_{2}, x_{1} y_{1}+x_{2} y_{2}+x_{3} y_{3}\right\rangle \\
\text { and } \mathcal{I}_{2(I)}=\left\langle x_{3},-h_{2} x_{1}+h_{1} x_{2}, x_{0} y_{0}+x_{1} y_{1}+x_{2} y_{2}\right\rangle \\
\mathcal{I}_{(I I)}=\mathcal{I}_{1(I I)} \cap \mathcal{I}_{2(I I)} \cap \mathcal{I}_{3(I I)}, \\
\text { where } \mathcal{I}_{1(I)}=\left\langle x_{0},-h_{2} x_{1}+h_{1} x_{2}, x_{1} y_{1}+x_{2} y_{2}+x_{3} y_{3}\right\rangle \\
\text { and } \mathcal{I}_{2(I)}=\left\langle x_{3}, h_{1} x_{1}+h_{2} x_{2}, x_{0} y_{0}+x_{1} y_{1}+x_{2} y_{2}\right\rangle
\end{gathered}
$$

where $h_{1}$ and $h_{2}$ are functions of $v_{13}$ and $v_{24}$. For instance, $\mathcal{I}_{1(I)}$ consists of

\begin{tabular}{lllllllll}
\hline$v_{13}$ & -1 & $-\frac{1}{2}$ & $-\frac{1}{4}$ & 0 & $\frac{1}{4}$ & $\frac{1}{2}$ & 1 & $w$ \\
\hline$h_{1}$ & -1 & -4 & -8 & 0 & 8 & 4 & 1 & $\frac{2}{w}, w \neq 0$ \\
$h_{2}$ & 0 & -3 & -15 & 0 & -15 & -3 & 0 & $1-\frac{1}{w^{2}}, w \neq 0$ \\
\hline
\end{tabular}

$x_{0}$ and $\left.\mathrm{S}_{6}^{2}\right|_{x_{0}=0}$ irrespective of the value of $v_{13}$. The remaining polynomial has coefficients $h_{1}$ and $h_{2}$, whose values are listed in Table 1 for arbitrarily chosen $v_{13}$ along with their interpolated values for a general $v_{13}=w$. Thus, Eq. (8) can be further simplified as follows:

$$
\begin{aligned}
\mathcal{I}_{1(I)} & =\left\langle x_{0}, 2 v_{13} x_{1}+\left(v_{13}^{2}-1\right) x_{2}, x_{1} y_{1}+x_{2} y_{2}+x_{3} y_{3}\right\rangle \\
\mathcal{I}_{2(I)} & =\left\langle x_{3},\left(1-v_{13}^{2}\right) x_{1}+2 v_{13} x_{2}, x_{0} y_{0}+x_{1} y_{1}+x_{2} y_{2}\right\rangle \\
\mathcal{I}_{1(I I)} & =\left\langle x_{0},\left(1-v_{24}^{2}\right) x_{1}+2 v_{24} x_{2}, x_{1} y_{1}+x_{2} y_{2}+x_{3} y_{3}\right\rangle \\
\mathcal{I}_{2(I I)} & =\left\langle x_{3}, 2 v_{24} x_{1}+\left(v_{24}^{2}-1\right) x_{2}, x_{0} y_{0}+x_{1} y_{1}+x_{2} y_{2}\right\rangle
\end{aligned}
$$

As a result, the first two operation modes of the 4-rRUU PM are:

$$
\begin{aligned}
\mathcal{I}_{1} & =\mathcal{I}_{1(I)} \cup \mathcal{I}_{1(I I)} \\
& =\left\langle x_{0}, 2 v_{13} x_{1}+\left(v_{13}^{2}-1\right) x_{2},\left(1-v_{24}^{2}\right) x_{1}+2 v_{24} x_{2}, x_{1} y_{1}+x_{2} y_{2}+x_{3} y_{3}\right\rangle \\
\mathcal{I}_{2} & =\mathcal{I}_{2(I)} \cup \mathcal{I}_{2(I I)} \\
& =\left\langle x_{3},\left(1-v_{13}^{2}\right) x_{1}+2 v_{13} x_{2}, 2 v_{24} x_{1}+\left(v_{24}^{2}-1\right) x_{2}, x_{0} y_{0}+x_{1} y_{1}+x_{2} y_{2}\right\rangle
\end{aligned}
$$

In general, $\mathcal{I}_{1}=\left\langle x_{0}, x_{1}, x_{2}, y_{3}\right\rangle$ and $\mathcal{I}_{2}=\left\langle x_{3}, x_{1}, x_{2}, y_{0}\right\rangle$. The former corresponds to a 3-dof pure translational mode, where the platform is upside down with the $z_{P}$-axis pointing downwards. The latter is also a 3 -dof translational mode, but 
the platform is in upright position with $z_{P}$-axis pointing upwards. An example would be the configuration (2) in [6] with $\beta_{i}=90^{\circ}, i=1,2,3,4$.

However, the set of binomial equations $2 v_{13} x_{1}+\left(v_{13}^{2}-1\right) x_{2}=0$ and $\left(1-v_{24}^{2}\right) x_{1}+$ $2 v_{24} x_{2}=0$ can have non-trivial values for $x_{1}$ and $x_{2}$ if the following conditions are satisfied:

$$
\begin{aligned}
\frac{2 v_{13}}{v_{13}^{2}-1}=\frac{1-v_{24}^{2}}{2 v_{24}} & \Longrightarrow \tan \left(\beta_{1}\right)=\tan \left(\beta_{3}\right)=-\cot \left(\beta_{2}\right)=-\cot \left(\beta_{4}\right) \\
& \Longrightarrow \beta_{1}=\beta_{3}=\beta_{2}-90^{\circ}=\beta_{4}-90^{\circ}
\end{aligned}
$$

In that case, the first two operation modes are

$$
\begin{aligned}
\mathcal{I}_{1} & =\mathcal{I}_{1(I)} \cup \mathcal{I}_{1(I I)} \\
& =\left\langle x_{0}, 2 v_{13} x_{1}+\left(v_{13}^{2}-1\right) x_{2}, x_{1} y_{1}+x_{2} y_{2}+x_{3} y_{3}\right\rangle \\
\mathcal{I}_{2} & =\mathcal{I}_{2(I)} \cup \mathcal{I}_{2(I I)} \\
& =\left\langle x_{3},\left(1-v_{13}^{2}\right) x_{1}+2 v_{13} x_{2}, x_{0} y_{0}+x_{1} y_{1}+x_{2} y_{2}\right\rangle .
\end{aligned}
$$

With $x_{3}=1$, the Study parameters corresponding to the first operation mode, $\mathcal{I}_{1}$ are $\left[0, x_{1}, x_{2}, 1, y_{0}, y_{1}, y_{2},-x_{1} y_{1}-x_{2} y_{2}\right]$. Thus, only four independent parameters are sufficient to characterize this operation mode and it corresponds to a 4-dof Schönflies mode in which the translational motions are parametrized by $y_{0}, y_{1}$ and $y_{2}$ and the rotational motion is parametrized by $x_{1}, x_{2}$ along with $2 v_{13} x_{1}+\left(v_{13}^{2}-1\right) x_{2}=\left(1-v_{24}^{2}\right) x_{1}+2 v_{24} x_{2}=0$. In this operation mode, the platform is upside down with the $z_{P}$-axis pointing in a direction opposite to the $z_{O}$-axis. The rotational motion is about an axis located at an angle of $\beta_{1}-90^{\circ}=\beta_{3}-90^{\circ}=\beta_{2}=\beta_{4}$ from the $x_{O}$-axis.

Similarly, with $x_{0}=1$, the Study parameters corresponding to the first operation mode, $\mathcal{I}_{2}$ are $\left[1, x_{1}, x_{2}, 0,-x_{1} y_{1}-x_{2} y_{2}, y_{1}, y_{2}, y_{3}\right]$. Thus, only four independent parameters are sufficient to characterize this operation mode and it is a 4-dof Schönflies mode in which the translational motions are parametrized by $y_{1}, y_{2}$ and $y_{3}$ and the rotational motion is parametrized by $x_{1}, x_{2}$ along with $\left(1-v_{13}^{2}\right) x_{1}+2 v_{13} x_{2}=2 v_{24} x_{1}+\left(v_{24}^{2}-1\right) x_{2}=0$. In this operation mode, the platform is in upright position with rotational motion about an axis located at an angle of $\beta_{1}-90^{\circ}=\beta_{3}-90^{\circ}=\beta_{2}=\beta_{4}$ from the $x_{O}$-axis.

Hence, it can be concluded from Eqs. (11) and (12) that when all the base Rjoint axes of the 4-rRUU PM have the same horizontal orientations, it exhibits a Schönflies motion mode with the rotational dof about a horizontal axis with the same orientation as those R-joint axes. An example is the configuration (1) in [6], where $\beta_{1}=\beta_{3}=90^{\circ}, \beta_{2}=\beta_{4}=0^{\circ}$.

\section{Workspace for the same orientations of base $\mathrm{R}$-joint axes}

The moving platform center of the 4-rRUU PM lies on the boundary of the translational workspace only when at least one of its limbs is in a fully extended 
or a folded configuration. In this case, the limb is said to be in a limb or serial or input singularity [8]. It can happen when the $8 \times 4$ input Jacobian matrix $\mathbf{J}_{I}=\frac{\partial g}{\partial t_{i}}$ with $i=1,2,3,4$ and $g=\left[g_{1}, g_{2}, \ldots, g_{8}\right]$ is not full-rank. One approach to deal with the non-square matrix is to search for conditions such that all its $4 \times 4$ minors vanish as shown for a 3-RUU PM in [9]. However, the first three equations of the 3-RUU PM do not depend on the variable $t_{i}$ which is the half tangent of the actuated joint variable $\theta_{i}$. Likewise, here, $g_{i}$ does not depend on $t_{i}, i=1,2,3,4$. Therefore, the input singularities corresponding to limb $i$ can be simply calculated as $f_{i}: \frac{\partial g_{i+4}}{\partial t_{i}}=0, i=1,2,3,4$. Eliminating $t_{i}$ from $f_{i}$ and $g_{i+4}$ leads to four polynomials, $S_{i}$ solely in terms of Study parameters. The singularity surfaces (tori shaped) are nothing but the varieties of these polynomials and the workspace boundary is given by their intersection. By considering the 4rRUU PMs in Schönflies motion mode, it is possible to visualize the translational workspace boundaries for different fixed orientations of the moving platform. For instance, the singularity surfaces of a 4-rRUU PM with base R-joint axes parallel to $x_{O}$-axis and with design parameters $r_{0}=2, r_{1}=1, p=2, q=3$ have the following implicit representations:

$$
\begin{aligned}
S_{1}: & x^{4}+2 x^{2} y^{2}+2 x^{2} z^{2}+y^{4}+2 y^{2} z^{2}+z^{4}-4 x^{3}-4 x y^{2}-4 x z^{2}-4 x^{2}-24 y^{2} \\
& -24 z^{2}+16 x+16=0 \\
S_{2}: & x^{4}+2 x^{2} y^{2}+2 x^{2} z^{2}+y^{4}+2 y^{2} z^{2}+z^{4}-4 x^{2} y-4 y^{3}-4 y z^{2}-8 x^{2}-20 y^{2} \\
& -24 z^{2}+48 y=0 \\
S_{3}: & x^{4}+2 x^{2} y^{2}+2 x^{2} z^{2}+y^{4}+2 y^{2} z^{2}+z^{4}+4 x^{3}+4 x y^{2}+4 x z^{2}-4 x^{2}-24 y^{2} \\
& -24 z^{2}-16 x+16=0 \\
S_{4}: & x^{4}+2 x^{2} y^{2}+2 x^{2} z^{2}+y^{4}+2 y^{2} z^{2}+z^{4}+4 x^{2} y+4 y^{3}+4 y z^{2}-8 x^{2}-20 y^{2} \\
& -24 z^{2}-48 y=0
\end{aligned}
$$

where the orientation of moving platform, $\phi=0^{\circ}$ and $(x, y, z)$ are the coordinates of point $P$. The workspace boundary for this PM is shown in Fig. 2c. Additionally, Fig. 2 shows the workspaces for three configurations of the PM with their base $\mathrm{R}$-joint axes parallel to $x_{O}, y_{O}$ and $z_{O}$-axes, henceforth named as 4- $R_{x} U U, 4-R_{y} U U$ and $4-R_{z} U U$ PMs, respectively. The workspaces are plotted for different orientations of the moving platform including their cross-sections about a symmetric axis a for the 4- $\mathrm{R}_{\mathrm{a}} \mathrm{UU} \mathrm{PM}$, where a can be $x, y$ or $z$.

\section{Conclusions and future work}

In this paper, a dual reconfigurable 4-rRUU parallel manipulator was considered to determine its operation modes as a function of the orientation of its base revolute joint axes. The constraint equations were derived using Study's kinematic mapping for some specific base R-joint orientations such that the coefficients are 


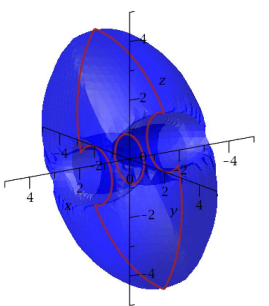

(a) $\phi_{x}=-90^{\circ}$

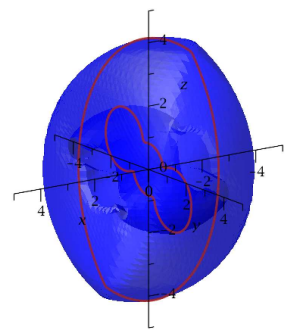

(d) $\phi_{x}=45^{\circ}$

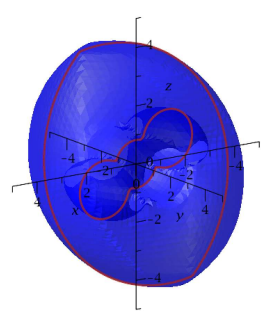

(g) $\phi_{y}=-45^{\circ}$

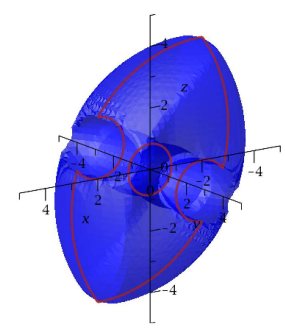

(j) $\phi_{y}=90^{\circ}$

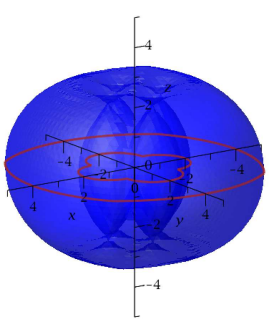

(m) $\phi_{z}=0^{\circ}$

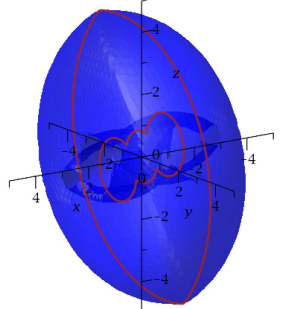

(b) $\phi_{x}=-45^{\circ}$

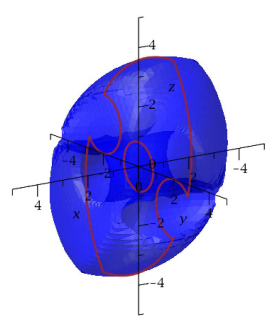

(e) $\phi_{x}=90^{\circ}$

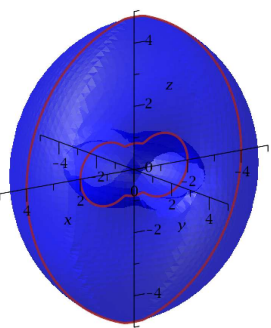

(h) $\phi_{y}=0^{\circ}$

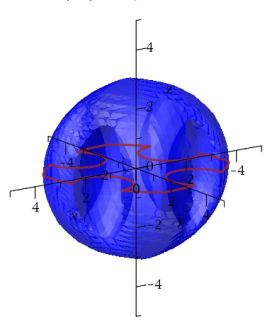

(k) $\phi_{z}=-90^{\circ}$

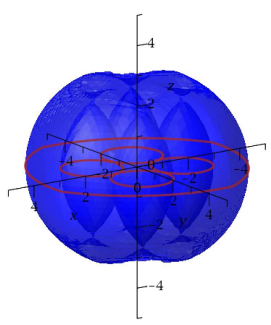

(n) $\phi_{z}=45^{\circ}$

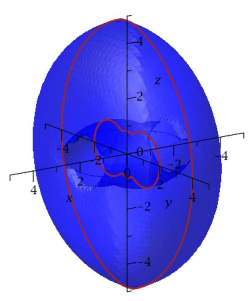

(c) $\phi_{x}=0^{\circ}$

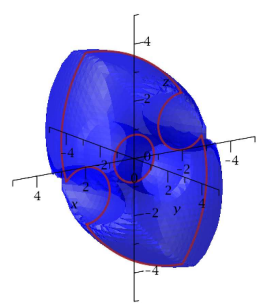

(f) $\phi_{y}=-90^{\circ}$

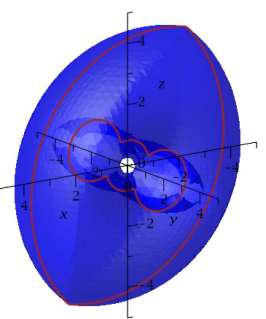

(i) $\phi_{y}=45^{\circ}$

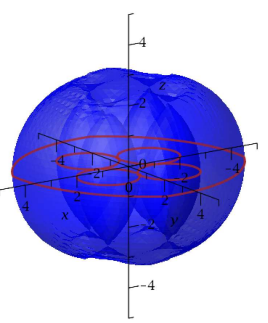

(l) $\phi_{z}=-45^{\circ}$

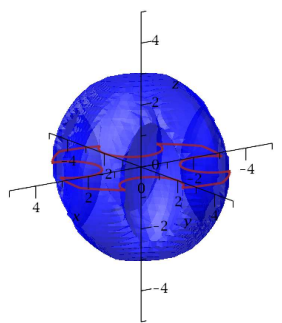

(o) $\phi_{z}=90^{\circ}$

Fig. 2: Workspaces of 4- $R_{x} U U(a-e), 4-R_{y} U U(f-j)$ and 4- $R_{z} U U(k-o) P M s$ 
always rational. It simplified the primary decomposition of the constraint equations to determine the operation modes. Then, the polynomials characterizing those operation modes were interpolated to obtain the operation modes for any base R-joint orientations. Furthermore, the translational workspace was plotted for three mutually perpendicular orientations of the base R-joints starting from the algebraic equations describing limb singularities.

As a part of the future work, design optimization of the PM under study will be performed to obtain optimal design parameters so as to realize a functional prototype.

\section{References}

1. Dimiter Zlatanov, Ilian A. Bonev, and Clément M. Gosselin. Constraint Singularities as C-Space Singularities, pages 183-192. Springer Netherlands, Dordrecht, 2002.

2. Dominic R Walter, Manfred L Husty, and Martin Pfurner. A complete kinematic analysis of the SNU 3-UPU parallel robot. Contemporary Mathematics, 496:331, 2009.

3. Dominic R Walter and Manfred L Husty. Kinematic analysis of the TSAI 3UPU parallel manipulator using algebraic methods. In The 13th IFToMMWorld Congress in Mechanism and Machine Science, Guanajuato, Mexico, pages 1-10, 2011.

4. J. Schadlbauer, D.R. Walter, M.L. Husty, J. Schadlbauer, D.R. Walter, and M.L. Husty. The 3-rps parallel manipulator from an algebraic viewpoint. Mechanism and Machine Theory, 75(Complete):161-176, 2014.

5. Latifah Nurahmi, Stéphane Caro, Philippe Wenger, Josef Schadlbauer, and Manfred Husty. Reconfiguration analysis of a 4-RUU parallel manipulator. Mechanism and Machine Theory, 96:269-289, 2016.

6. Abhilash Nayak, Stéphane Caro, and Philippe Wenger. A Dual Reconfigurable 4rRUU Parallel Manipulator. In The 4th IEEE/IFToMM International conference on Reconfigurable Mechanisms and Robots (ReMAR2018), Delft, Netherlands, June 2018.

7. David A. Cox, John Little, and Donal O'Shea. Ideals, Varieties, and Algorithms: An Introduction to Computational Algebraic Geometry and Commutative Algebra. Springer, 3rd edition, 2007.

8. C. Gosselin and J. Angeles. Singularity analysis of closed-loop kinematic chains. IEEE Transactions on Robotics and Automation, 6(3):281-290, June 1990.

9. Thomas Stigger, Martin Pfurner, and Manfred Husty. Workspace and singularity analysis of a 3-RUU parallel manipulator. In Burkhard Corves, Philippe Wenger, and Mathias Hüsing, editors, EuCoMeS 2018, pages 325-332, Cham, 2019. Springer International Publishing.

10. David H. Eberly. Chapter 15 - intersection methods. In David H. Eberly, editor, $3 D$ Game Engine Design (Second Edition), The Morgan Kaufmann Series in Interactive 3D Technology, pages 681 - 717. Morgan Kaufmann, San Francisco, second edition edition, 2007. 